\title{
An Adaptive Sliding Mode Control Law for the Power Maximization of the Wind Turbine System
}

\author{
Oscar Barambones and Jose Maria Gonzalez de Durana \\ Automatic Control and System Engineering Departament \\ EUI de Vitoria. University of the Basque Country \\ Nieves cano 12. 1006 Vitoria. (SPAIN) \\ oscar.barambones@ehu.es
}

\begin{abstract}
The actual wind turbines are provided with adjustable speed generators, like the double feed induction generator, that are capable to work in variable speed operations. One of the main advantage of adjustable speed generators is that they improve the system efficiency compared to fixed speed generators because turbine speed is adjusted as a function of wind speed to maximize output power. However this systems requires a suitable speed controller in order to track the optimal wind turbine reference speed. In this work, an adaptive robust control for variable speed wind power generator is described. The proposed robust control law is based on a sliding mode control theory, that presents a good performance under system uncertainties. The proposed sliding-mode control law incorporates an adaptive switching gain, which avoids having to calculate an upper limit of the system uncertainties that is necessary in the traditional sliding-mode control laws.

The stability analysis of the proposed controller under disturbances and parameter uncertainties is provided using the Lyapunov stability theory. Finally simulated results show, on the one hand that the proposed controller provides high-performance dynamic characteristics, and on the other hand that this scheme is robust with respect to plant parameter variations and external disturbances.
\end{abstract}

\section{INTRODUCTION}

Wind energy is an abundant renewable source of electricity by converting the kinetic energy of moving air mass into electricity. One of the most popular wind energy conversion system is the variable-speed wind turbines (VSWT), because this wind turbine are capable to track the changes in the wind speed by adapting shaft speed and thus maintaining optimal power generation. As it is well known the behavior of the VSWT is significantly affected by the control strategy used [1]. The main control objective of VSWT is power efficiency maximization. To reach this goal, the generator speed should be regulated in order to maintain the turbine tip speed ratio at its optimum value despite wind speed variations [2].

This paper investigates a new robust adaptive speed control method for variable speed wind turbines with Double Fed Induction Generator (DFIG) [3], [4]. The objective is to make the rotor speed track the desired speed in spite of system uncertainties in order to maximize the electric power generation. This is achieved by regulating the rotor current of the DFIG using the sliding mode control theory. However the traditional sliding control schemes requires the prior knowledge of the upper bound for the system uncertainties because this bound is employed to calculate the switching gain. Moreover, this upper bound should be determined as precisely as possible, because as higher is the upper bound higher value should be considered for the switching gain, and therefore the control effort will also be high. This is undesirable in practice because implies higher control signals and increases the chattering phenomenon.

In order to surmount this drawback, the presented paper proposes an adaptive law to calculate the switching gain. This adaptive law avoids the necessity of calculate an upper bound for the system uncertainties.

\section{SySTEM MODELLING}

The power extraction of wind turbine is a function of three main factors: the wind power available, the power curve of the machine and the ability of the machine to respond to wind fluctuation. The expression for power produced by the wind is given by [5]:

$$
P_{m}(v)=\frac{1}{2} C_{p}(\lambda, \beta) \rho \pi R^{2} v^{3}
$$

where $\rho$ is air density, $R$ is radius of rotor, $v$ is wind speed, $C_{p}$ denotes power coefficient of wind turbine, $\lambda$ is the tipspeed ratio and $\beta$ represents pitch angle. The tip-speed ratio is defined as

$$
\lambda=\frac{R w}{v}
$$

where $w$ is the turbine rotor speed. Therefore, if the rotor speed is kept constant, then any change in the wind speed will change the tip-speed ratio, leading to the change of power coefficient $C_{p}$, as well as the generated power out of the wind turbine. However, if the rotor speed is adjusted according to the wind speed variation, then the tip-speed ratio can be maintained at an optimal point, which could yield maximum power output from the system.

From equations 1 and 2 it is deduced that the input wind torque is:

$$
T_{m}(v)=\frac{P_{m}(v)}{w}=\frac{P_{m}(v)}{\frac{\lambda v}{R}}=k_{v} \cdot v^{2}
$$

where

$$
k_{v}=\frac{1}{2} C_{p} \rho \pi \frac{R^{3}}{\lambda}
$$


For a typical wind power generation system, the following simplified elements are used to illustrate the fundamental work principle. The system primarily consists of an aeroturbine, which converts wind energy into mechanical energy, a gearbox, which serves to increase the speed and decrease the torque and a generator to convert mechanical energy into electrical energy.

Driving by the input wind torque $T_{m}$, the rotor of the wind turbine runs at the speed $w$. The transmission output torque is then fed to the generator, which produces a shaft torque of $T_{e}$ at generator angular velocity of $w_{e}$. Note that the rotor speed and generator speed are not the same in general, due to the use of the gearbox.

The mechanical equations of the system can be characterized by [6]:

$$
J \dot{w}+B w=T_{m}-\gamma T_{e}
$$

where $J$ is the equivalent inertia moment of the turbine and the generator, $B$ and is the equivalent viscous friction coefficient of the the turbine and the generator, $T_{m}$ is the wind generated torque in the turbine, $T_{e}$ is the the generator torque, $w$ is the angular velocity of the turbine shaft, $w_{e}$ is the angular velocity of the generator rotor and $\gamma=\frac{w_{e}}{w}$ is the gear ratio.

Now we are going to consider the system electrical equations. In this work it is used a double feed induction generator. This induction machine is feed from both stator and rotor sides. The stator is directly connected to the grid while the rotor is fed through a variable frequency converter (VFC). In order to produce electrical active power at constant voltage and frequency to the utility grid, over a wide operation range (from subsynchronous to supersynchronous speed), the active power flow between the rotor circuit and the grid must be controlled both in magnitude and in direction. Therefore, the VFC consists of two four-quadrant IGBT PWM converters (rotor-side converter and grid-side converter) connected backto-back by a dc-link capacitor [7], [8].

The DFIG can be regarded as a traditional induction generator with a nonzero rotor voltage. Using the stator-flux oriented reference frame, the $\mathrm{d}$-axis is aligned with the stator flux linkage vector $\psi_{s}$, and then, $\psi_{d s}=\psi_{s}$ and $\psi_{q s}=0$. This yields the following relationships [10]:

$$
\begin{aligned}
i_{q s} & =\frac{L_{m} i_{q r}}{L_{s}} \\
i_{d s} & =\frac{L_{m}\left(i_{m s}-i_{d r}\right)}{L_{s}} \\
i_{m s} & =\frac{v_{q s}-r_{s} i_{q s}}{w_{s} L_{m}} \\
T_{e} & =\frac{-L_{m} i_{m s} i_{q r}}{L_{s}} \\
Q_{s} & =\frac{3}{2} \frac{w_{s} L_{m}^{2} i_{m s}\left(i_{m s}-i_{d r}\right)}{L_{s}} \\
v_{d r} & =r_{r} i_{d r}+\sigma L_{r} \frac{d i_{q r}}{d t}-s w_{s} \sigma L_{r} i_{q r} \\
v_{q r} & =r_{r} i_{q r}+\sigma L_{r} \frac{d i_{q r}}{d t}
\end{aligned}
$$

$$
+s w_{s}\left(\frac{\sigma L_{r} i_{d r}+L_{m}^{2} i_{m s}}{L_{s}}\right)
$$

where $w_{s}$ is the rotational speed of the synchronous reference frame, $s w_{s}=w_{s}-w_{e}$ is the slip frequency, $w_{e}$ is the generator rotor speed, $L_{s}, L_{r}$, and $L_{m}$ are the stator inductance, rotor inductance and mutual inductances, respectively and $\sigma=1-$ $\frac{L_{m}^{2}}{L_{s} L_{r}}$.

Since the stator is connected to the grid, and the influence of the stator resistance is small, the stator magnetizing current $i_{m s}$ can be considered constant [7]. Therefore, the electromagnetic torque can be defined as follows:

$$
T_{e}=-K_{T} i_{q r}
$$

where $K_{T}$ is a torque constant, and is defined as follows:

$$
K_{T}=\frac{L_{m} i_{m s}}{L_{s}}
$$

\section{DFIG CONTROL SCHEME}

The DFIG wind turbine control system generally consists of two parts: the electrical control on the DFIG and the mechanical control on the wind turbine blade pitch angle. Control of the DFIG is achieved controlling the variable frequency converter, which includes control of the rotor-side converter (RSC) and control of the grid-side converter (GSC). The objective of the RSC is to govern both the stator-side active and reactive powers independently; while the objective of the GSC is to keep the dc-link voltage constant regardless of the magnitude and direction of the rotor power. The GSC control scheme can also be designed to regulate the reactive power or the stator terminal voltage of the DFIG.

When the wind turbine generator (WTG) operates in the variable-speed mode, in order to extract the maximum active power from the wind, the shaft speed of the WTG must be adjusted to achieve an optimal tip-speed ratio $\lambda_{\text {opt }}$, which yields the maximum power coefficient $C_{p_{\max }}$, and therefore the maximum power. In other words, given a particular wind speed, there is a unique wind turbine speed required to achieve the goal of maximum wind power extraction. The value of the $\lambda_{\text {opt }}$ can be calculated from the maximum of the power coefficient curves versus tip-speed ratio, which depends of the modeling turbine characteristics.

The power coefficient $C_{p}$, can be approximated by equation 16 based on the modeling turbine characteristics [9]:

$$
C_{p}(\lambda, \beta)=c_{1}\left(\frac{c_{2}}{\lambda_{i}}-c_{3} \beta-c_{4}\right) e^{\frac{-c_{5}}{\lambda_{i}}}+c_{6} \lambda
$$

where the coefficients $c_{1}$ to $c_{6}$ depends on the wind turbine design characteristics, and $\lambda_{i}$ is defined as

$$
\frac{1}{\lambda_{i}}=\frac{1}{\lambda+0.08 \beta}-\frac{0.035}{\beta^{3}+1}
$$

The value of $\lambda_{\text {opt }}$ can be calculated from the roots of the derivative of the equation 16. Then, based on the wind 
speed, the corresponding optimal generator speed command for maximum wind power tracking is determined by:

$$
w^{*}=\frac{\lambda_{o p t} \cdot v}{R}
$$

\section{SPEED CONTROLLER DESIGN}

A suitably designed speed controller is essential to track the optimal wind turbine speed reference $w^{*}$ for maximum wind power extraction. The design of such a speed controller should take into account the dynamics of the WTG shaft system.

\section{A. Rotor Side Converter Control}

The RSC control scheme is expected to achieve the following objectives:

1) Regulating the DFIG rotor speed for maximum wind power capture.

2) Maintaining the DFIG stator output voltage frequency constant.

3) Controlling the DFIG reactive power.

In the DFIG-based wind generation system, these objectives are commonly achieved by electrical generator rotor current regulation on the stator-flux oriented reference frame. From equations 5 and 14 it is deduced that the wind turbine speed can be controlled by regulating the q-axis rotor current components $\left(i_{q r}\right)$ while equation 10 indicates that the stator reactive power $\left(Q_{s}\right)$ can be controlled by regulating the d-axis rotor current components, $\left(i_{d s}\right)$. Consequently, the reference values of $i_{q r}$ and $i_{d r}$ can be determined directly from $w_{r}$ and $Q_{s}$ references.

Then, from equations 5 and 14 the dynamic equation of the system is obtained:

$$
\begin{aligned}
\dot{w} & =\frac{1}{J}\left(T_{m}-\gamma K_{T} i_{q r}-B w\right) \\
& =-a w+f-b i_{q r}
\end{aligned}
$$

where the parameters are defined as:

$$
a=\frac{B}{J}, \quad b=\frac{\gamma K_{T}}{J}, \quad f=\frac{T_{m}}{J} ;
$$

Now, we are going to consider the previous mechanical equation (20) with uncertainties as follows:

$$
\dot{w}=-(a+\triangle a) w+(f+\triangle f)-(b+\triangle b) i_{q s}
$$

where the terms $\triangle a, \triangle b$ and $\triangle f$ represents the uncertainties of the terms $a, b$ and $f$ respectively.

Let us define define the speed tracking error as follows:

$$
e(t)=w(t)-w^{*}(t)
$$

where $w^{*}$ is the rotor speed command that provides the maximum wind power extraction.

Taking the derivative of the previous equation with respect to time yields:

$$
\dot{e}(t)=\dot{w}-\dot{w}^{*}=-a e(t)+u(t)+d(t)
$$

where the following terms have been collected in the signal $u(t)$,

$$
u(t)=f(t)-b i_{q r}(t)-a w^{*}(t)-\dot{w}^{*}(t)
$$

and the uncertainty terms have been collected in the signal $d(t)$,

$$
d(t)=-\triangle a w(t)+\triangle f(t)-\triangle b i_{q r}(t)
$$

In order to compensate the above described system uncertainties, an adaptive sliding control scheme is proposed. In the sliding control theory, the switching gain must be constructed so as to attain the sliding condition [11]. In order to meet this condition a suitable choice of the switching gain should be made to compensate for the uncertainties.

The sliding variable $S(t)$ is defined with an integral component in order to avoid the speed tracking error calculation:

$$
S(t)=e(t)+\int_{0}^{t}(k+a) e(\tau) d \tau
$$

where $k$ is a constant gain.

Then the sliding surface is defined as:

$$
S(t)=e(t)+\int_{0}^{t}(a+k) e(\tau) d \tau=0
$$

Now, we are going to design a variable structure speed controller, that incorporates an adaptive switching gain, in order to control the wind turbine speed.

$$
u(t)=-k e(t)-\hat{\phi}(t) \gamma \operatorname{sgn}(S)
$$

where the $k$ is the gain defined previously, $\hat{\phi}$ is the estimated switching gain, $\gamma$ is a positive constant, $S$ is the sliding variable defined in eqn. (27) and $\operatorname{sgn}(\cdot)$ is the signum function.

The switching gain $\hat{\phi}$ is adapted according to the following updating law:

$$
\dot{\hat{\phi}}(t)=\gamma|S(t)| \quad \hat{\phi}(0)=0
$$

where $\gamma$ is a positive constant that let us choose the adaptation speed for the switching gain.

In order to obtain the speed trajectory tracking, the following assumptions should be formulated:

$(\mathcal{A} 1)$ The gain $k$ must be chosen so that the term $(k+a)$ is strictly positive, therefore the constant $k$ should be $k>-a$.

$(\mathcal{A} 2)$ There exits an unknown finite non-negative switching gain $\phi$ such that

$$
\phi>d_{\max }+\eta \quad \eta>0
$$

where $d_{\max } \geq|d(t)| \forall t$ and $\eta$ is a positive constant.

Note that this condition only implies that the uncertainties of the system are bounded magnitudes, but the knowledge of this bound is not required.

(A3) The constant $\gamma$ must be chosen so that $\gamma \geq 1$. 
Theorem 1: Consider the induction motor given by equation (22). Then, if assumptions $(\mathcal{A} 1),(\mathcal{A} 2)$ and $(\mathcal{A} 3)$ are verified, the control law (29) and the adaptation law (30) leads the wind turbine speed $w(t)$, so that the speed tracking error $e(t)=w(t)-w^{*}(t)$ tends to zero as the time tends to infinity.

The proof of this theorem will be carried out using the Lyapunov stability theory.

Proof: Define the Lyapunov function candidate:

$$
V(t)=\frac{1}{2} S(t) S(t)+\frac{1}{2} \tilde{\phi}(t) \tilde{\phi}(t)
$$

where $S(t)$ is the sliding variable defined previously and $\tilde{\phi}(t)=\hat{\phi}(t)-\phi$

Its time derivative is calculated as:

$$
\begin{aligned}
\dot{V}(t) & =S(t) \dot{S}(t)+\tilde{\phi}(t) \dot{\tilde{\phi}}(t) \\
& =S \cdot[\dot{e}+(a+k) e]+\tilde{\phi}(t) \dot{\hat{\phi}}(t) \\
& =S \cdot[(-a e+u+d)+(k e+a e)]+\tilde{\phi} \gamma|S| \\
& =S \cdot[u+d+k e]+(\hat{\phi}-\phi) \gamma|S| \\
& =S \cdot[-k e-\hat{\phi} \gamma \operatorname{sgn}(S)+d+k e]+(\hat{\phi}-\phi) \gamma|S| \\
& =S \cdot[d-\hat{\phi} \gamma \operatorname{sgn}(S)]+\hat{\phi} \gamma|S|-\phi \gamma|S| \\
& =d S-\hat{\phi} \gamma|S|+\hat{\phi} \gamma|S|-\phi \gamma|S| \\
& \leq|d||S|-\phi \gamma|S| \\
& \leq|d||S|-\left(d_{\max }+\eta\right) \gamma|S| \\
& =|d||S|-d_{\max } \gamma|S|-\eta \gamma|S| \\
& \leq-\eta \gamma|S|
\end{aligned}
$$

then

$$
\dot{V}(t) \leq 0
$$

It should be noted that in the proof the equations (27), (24), (29) and (30), and the assumptions $(\mathcal{A} 2)$ and $(\mathcal{A} 3)$ have been used.

Using the Lyapunov's direct method, since $V(t)$ is clearly positive-definite, $\dot{V}(t)$ is negative definite and $V(t)$ tends to infinity as $S(t)$ tends to infinity, then the equilibrium at the origin $S(t)=0$ is globally asymptotically stable. Therefore $S(t)$ tends to zero as the time tends to infinity. Moreover, all trajectories starting off the sliding surface $S=0$ must reach it in finite time and then will remain on this surface. This system's behavior once on the sliding surface is usually called sliding mode [11].

When the sliding mode occurs on the sliding surface (28), then $S(t)=\dot{S}(t)=0$, and therefore the dynamic behavior of the tracking problem (24) is equivalently governed by the following equation:

$$
\dot{S}(t)=0 \quad \Rightarrow \quad \dot{e}(t)=-(k+a) e(t)
$$

Then, under assumption $(\mathcal{A} 1)$, the tracking error $e(t)$ converges to zero exponentially.

It should be noted that, a typical motion under sliding mode control consists of a reaching phase during which trajectories starting off the sliding surface $S=0$ move toward it and reach it in finite time, followed by sliding phase during which the motion will be confined to this surface and the system tracking error will be represented by the reduced-order model (35), where the tracking error tends to zero.

Finally, the torque current command, $i_{q r}^{*}(t)$, can be obtained from equations (25) and (29):

$$
i_{q r}^{*}(t)==\frac{1}{b}\left[k e-\hat{\phi} \gamma \operatorname{sgn}(S)+a w_{m}^{*}+\dot{w}_{m}^{*}+f\right]
$$

Therefore, the proposed adaptive sliding mode control resolves the speed tracking problem for the wind turbine power maximization in the presence of system uncertainties. Moreover, this wind turbine speed tracking let us obtain the maximum wind power extraction for all wind speed values that appears along the time.

\section{B. Grid Side Converter Control}

The objective of the GSC control is to keep the dc link voltage constant regardless of the direction of rotor power flow. A vector control approach is used, with a reference frame oriented along the stator (or supply) voltage vector position. In such scheme, direct axis current is controlled to keep the dc link voltage constant and quadrature axis current component can be used to regulate the reactive power flow between the supply side converter and the supply. All voltage and current quantities are transformed to a reference frame that rotates at the same speed as the supply voltage space phase with the real axis (d-axis) of the reference frame aligned to the supply voltage vector. At steady state, the reference frame speed equals the synchronous speed.

The scheme makes use of the supply voltage angle determined dynamically to map the the supply voltage, the converter terminal voltage and the phase currents onto the new reference frame.

In the stator voltage oriented reference frame, the d-axis is aligned to the supply voltage phasor $V_{s}$, and then $v_{d}=V_{s}$ and $v_{q}=0$. Hence, the powers between the grid side converter and the grid are:

$$
\begin{aligned}
P & =\frac{3}{2}\left(v_{d} i_{d}+v_{q} i_{q}\right)=\frac{3}{2} v_{d} i_{d} \\
Q=\frac{3}{2}\left(v_{q} i_{d}-v_{d} i_{q}\right) & =-\frac{3}{2} v_{d} i_{q}
\end{aligned}
$$

where $v_{d}$ and $v_{q}$ are the direct and quadrature components of the supply voltages, and $i_{d}$ and $i_{q}$ are the direct and quadrature components of the stator side converter input currents.

From the previous equations it is observed that the active and reactive power flow between supply side converter and the supply will be proportional to $i_{d}$ and $i_{q}$ respectively.

The dc power change has to be equal to the active power flowing between the grid and the grid side converter. Thus,

$$
\begin{aligned}
E i_{0 s} & =\frac{3}{2} v_{d} i_{d} \\
C \frac{d E}{d t} & =i_{0 s}-i_{0 r}
\end{aligned}
$$


where $i_{0 s}$ is the current between the dc link and the rotor and $i_{0 r}$ is the current between the dc link and the stator.

Therefore from eqn. (39) and (40) is concluded that the dc link voltage can be controlled using the current $i_{d}$. The control scheme utilizes a current control loop with the $i_{d}$ demand being derived from the dc-link voltage error through a standard PI controller.

\section{Simulation Results}

In this section we will study the speed regulation performance of the proposed adaptive sliding-mode field oriented control scheme. The objective of this regulation is to maximize the wind power extraction in order to obtain the maximum electrical power. In this sense, the wind turbine speed must be adjusted continuously against the wind speed variations.

The simulation are carried out using the Matlab/Simulink software and the turbine model is the one provided in the SimPowerSystems library [12].

In this example simulation it is used a variable speed wind farm with a rated power of $9 \mathrm{MW}$. The farm consists of six 1.5 MW wind turbines connected to a $575 \mathrm{~V}$ bus line. The wind turbines use a doubly-fed induction generator (DFIG) consisting of a wound rotor induction generator and an AC/DC/AC IGBT-based PWM converter. The stator winding is connected directly to the $60 \mathrm{~Hz}$ grid while the rotor is fed at variable frequency through the $\mathrm{AC} / \mathrm{DC} / \mathrm{AC}$ converter.

The system has the following mechanical parameters. The combined generator and turbine inertia constant is $J=5.04 \mathrm{~s}$ expressed in seconds, the combined viscous friction factor $B=0.01 p u$ in per-unit system (pu), based on the generator rating and there are 3 pole pairs [12].

In this simulation examples it is assumed that there is an uncertainty around $20 \%$ in the system parameters, that will be overcome by the proposed sliding control.

Finally, the following values have been chosen for the controller parameters, $k=1, \gamma=30$.

In this case study, the rotor is running at subsynchronous speed for wind speeds lower than $10 \mathrm{~m} / \mathrm{s}$ and it is running at a super-synchronous speed for higher wind speeds. In figure 1 , the turbine mechanical power as function of turbine speed is displayed in for wind speeds ranging from $5 \mathrm{~m} / \mathrm{s}$ to 16.2 $\mathrm{m} / \mathrm{s}$.

In this simulation it is used a variable wind speed, and figure 2 shows the wind speed used in this simulation.

Figure 3 show the reference (dashed line) and the real generator speed (solid line). As it may be observed, after a transitory time in which the switching gain is adapted and the sliding mode is reached, the generator speed tracks the desired speed in spite of system uncertainties. In this figure, the speed is expressed in the per unit system (pu), that is based in the generator synchronous speed $w_{s}=125.60 \mathrm{rad} / \mathrm{s}$.

However, at time $t=13 \mathrm{~s}$ a little speed error can be observed. This error appears because the wind torque uncertainty value is increased from $20 \%$ to $30 \%$ at this time, and then the control system lost the so called sliding mode because the

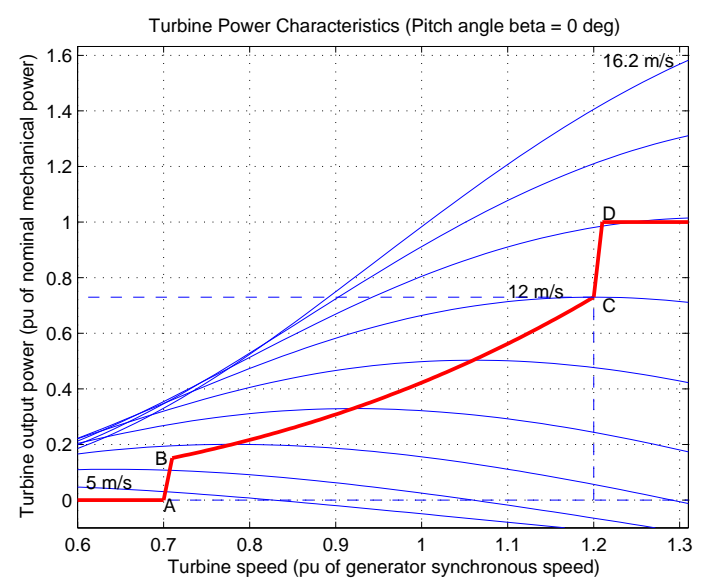

Fig. 1. Turbine Power Characteristics

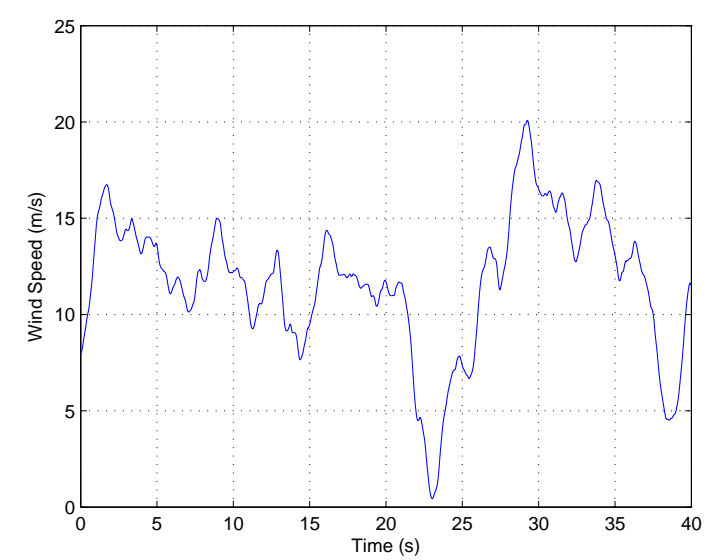

Fig. 2. Wind speed

actual sliding gain value is too small to overcome the new uncertainty introduced in the system due to the new higher wind torque uncertainty value. But then, after a small time the switching gain is adapted again so that this gain can compensate the new system uncertainties and so the generator speed error is eliminated.

Figure 4 shows the generated active power, whose value is maximized by our proposed adaptive sliding mode control scheme. As it can be observed in this figure, at time $32 \mathrm{~s}$ the mechanical power (and therefore the generated active power) should be limited by the pitch angle so as not to exceed the rated power, in order to prevent damages in the the system.

Figure 5 presents the time evolution of the estimated switching gain. The switching gain value starts from zero and then it is increased until its value is high enough to compensate for the system uncertainties. Then from time $2.5 \mathrm{~s}$ the sliding gain is remained constant because the system uncertainties remain constant as well. Later at time $13 \mathrm{~s}$, there is an increment in the system uncertainties and therefore the sliding gain is adapted once again in order to overcome the new system uncertainties. 


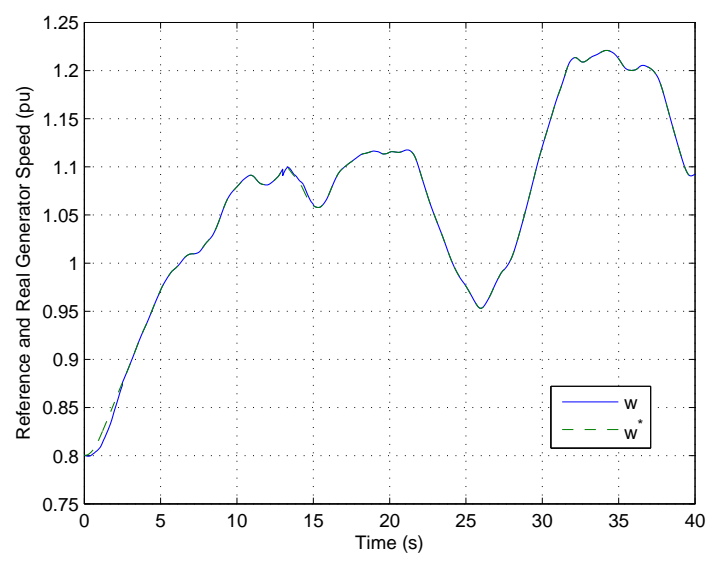

Fig. 3. Reference and real rotor speed

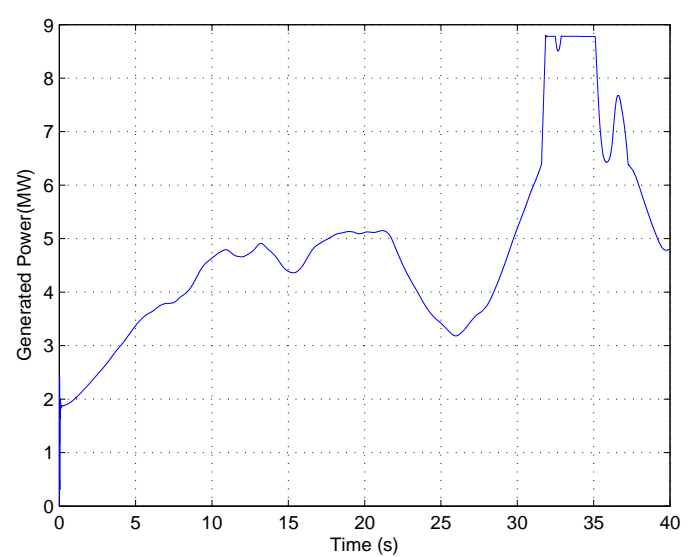

Fig. 4. Generated active power

\section{CONCLUSION}

In this paper an adaptive sliding mode vector control for a doubly feed induction generator, used in variable speed wind power generation is described. The proposed variable structure control has an integral sliding surface in order to relax the requirement of the acceleration signal, that is usual in conventional sliding mode speed control schemes. Due to the nature of the sliding control this control technique is robust under uncertainties that appear in the real systems. Therefore, the implemented control method allows the turbine to operate with the optimum power efficiency over a wide range of wind speed in the presence of system uncertainties. At wind speeds less than the rated wind speed, the speed controller seeks to maximize the power according to the maximum coefficient curve. As result, the variation of the generator speed follows the slow variation in the wind speed. At large wind speeds, the power limitation controller sets the blade angle to maintain rated power in order to prevent the system damages.

Finally, by means of simulation examples, it has been shown that the proposed design successfully controls the variable speed wind turbine system because the speed tracking

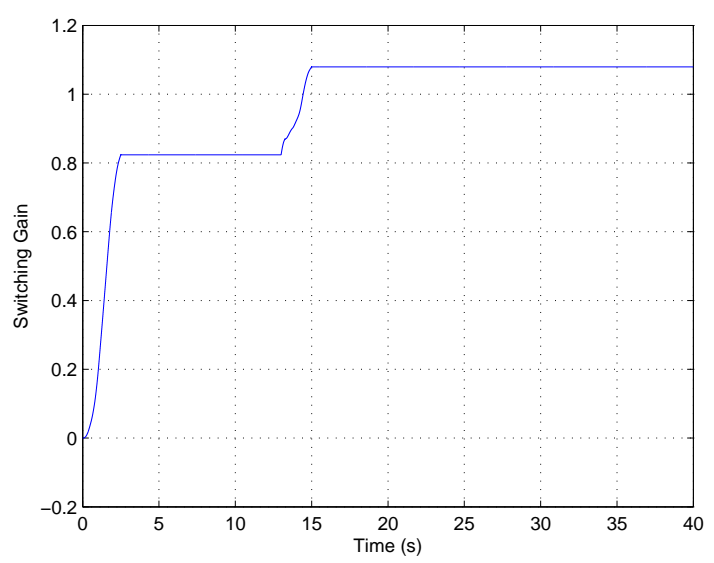

Fig. 5. Estimated switching gain

objective in order to get the maximum power extraction is achieved under system uncertainties.

\section{ACKNOWLEDGMENT}

The authors are very grateful to the Basque Government by the support of this work through the project S-PE09UN12 and to the UPV/EHU by its support through project GUI07/08 and GUI10/01.

\section{REFERENCES}

[1] B. Beltran, T. Ahmed-Ali, and M. H. Benbouzid, 2009, High-Order Sliding-Mode Control of Variable-Speed Wind Turbines, IEEE Trans. Indus. Electro., 56, 3314-3321.

[2] Xiyun Yang and Xinran Liu, 2010, Integral variable structure fuzzy adaptive control for variable speed wind power system, Int. Conf. on Logistics Systems and Intelligent Management, Harbin, China 2010, vol. 2, pp. 1247-1250.

[3] Muller, S., Deicke, M., de Doncker, R.W., 2002, Doubly fed induction generator system for wind turbines, IEEE Industry Application Magazine., 8, pp. 26-33.

[4] Bati, Akram, Rashid, Kasim, Al-Rubaiee, Safa. A New Methodology to the Control Problem of Horizontal Axis Wind Power Plants Using Adaptive Neural Proceedings of the 17th IFAC World Congress, 2008.

[5] Fernando D. Bianchi, Hrnan De Battista and Ricardo J. Mantz 2007 Wind Turbine Control Systems Advances in Industrial Control Series. Springer.

[6] Y.D. Song, B. Dhinakaran, X.Y. Bao, 2000, Variable speed control of wind turbines using nonlinear and adaptive algorithms, Journal of Wind Engineering and Industrial Aerodynamics., 85, pp. 293-308.

[7] R. Pena, J. C. Clare, and G. M. Asher 1996 Doubly fed induction generator using back-to-back PWM converters and its application to variablespeed wind-energy generation Proc. Inst. Elect. Eng., vol. 143, no. 3, pp. 231-241.

[8] Wei Qiao, Wei Zhou, Jos M. Aller, and Ronald G. Harley 2008, Wind Speed Estimation Based Sensorless Output Maximization Control for a Wind Turbine Driving a DFIG IEEE Trans. on Power Electronics, vol. 23 , no. 3, pp. 1156-1169.

[9] Siegfried Heier 1998 Grid Integration of Wind Energy Conversion Systems John Wiley \& Sons Ltd, 1998, ISBN 0-471-97143-X.

[10] Yazhou Lei, Alan Mullane, Gordon Lightbody, and Robert Yacamini 2006, Modeling of the Wind Turbine With a Doubly Fed Induction Generator for Grid Integration Studies, IEEE Trans. on Energy Conversion, vol. 21, no. 1, pp. 257-264.

[11] Utkin V.I., 1993, Sliding mode control design principles and applications to electric drives, IEEE Trans. Indus. Electro., 40, 26-36.

[12] SimPowerSystems 5 Users Guide The MathWorks 